УДК 004.89:65.011.56

3. Х. Борукаев, К. Б. Остапченко, О. И. Лисовиченко

\title{
ПОДХОД К ПОСТРОЕНИЮ СИСТЕМ ПОДДЕРЖКИ ПРИНЯТИЯ РЕШЕНИЙ ДЛЯ АВТОМАТИЗАЦИИ ПРОЦЕССОВ ОРГАНИЗАЦИОННОГО УПРАВЛЕНИЯ ЭНЕРГОРЫНКОМ
}

Аннотация: Приведены результаты исследования выбора и особенностей применения систем поддержки принятия решений для автоматизации процессов реализации функциональных задач организационного управления на оптовом рынке электроэнергии.

Ключевые слова: автоматизация, организационное управление, оптовый рынок электроэнергии, система поддержки принятия решений.

\section{Введение}

В современных условиях постоянно меняющихся цен на рынках энергоносителей существенно возрастает роль компьютерного моделирования динамики функционирования Оптового рынка электроэнергии (ОРЭ) для определения сбалансированной прогнозной оптовой цены покупки электроэнергии. В работе [1] приведена формулировка такой задачи математического и компьютерного моделирования, а в работах [2,3] проведено расчетно-экспериментальное исследование предложенных для ее решения моделей, их адекватности для краткосрочного прогнозирования оптовой цены покупки электроэнергии на ОРЭ. В результате анализа взаимосвязи данных динамики цен на электроэнергию и на энергоносители в [4], обоснована необходимость создания компьютерной системы мониторинга динамики энергорынка для решения ряда функциональных задач организационного управления ОРЭ.

В работе [5] отмечалось, что создание подобной системы является наукоёмкой и ресурсоёмкой задачей. Поэтому выбор и обоснование наиболее экономичного пути её решения на всех этапах от постановки задачи, проектирования, практической реализации, до непосредственного внедрения и последующей эксплуатации в течение возможно более продолжительного жизненного цикла, имеет весьма существенное значение.

Целью настоящей работы является дальнейшее обобщение и развитие предложенного в работе [5] подхода к построению компьютерных

\footnotetext{
๑ З. Х. Борукаев, К. Б. Остапченко, О. И. Лисовиченко
} 
систем организационного управления (КСОУ) на базе информационноаналитической системы мониторинга (ИАСМ), а также выбор и обоснование перехода к системе поддержки принятия решений (СППР) и включения в ее состав ИАСМ для построения интегрированной системы, ориентированной на исследование основных показателей состояния производства и экономики субъектов ОРЭ.

\section{1. Постановка задачи исследования}

Одним из способов организационного управления, получившим довольно широкое распространение при управлении сложными организационно-техническими системами, является ситуационное управление - управление на основе сложившихся фактов и обстоятельств, которые могут быть представлены в виде некоторой совокупности показателей функционирования объекта управления, его системы организационного управления и внешней среды их существования [6].

Формализация такого способа управления предполагает определение и введение понятий ситуации, её классификации и идентификации в некотором пространстве выходных показателей состояния управляемого объекта. При его реализации в СППР, которые предназначены для автоматизированного управления сложными системами, возникают определённые трудности. Они связаны с тем, что описание ситуаций и их идентификация с помощью множества показателей может оказаться невозможным за реальное время ввиду того, что число возможных сочетаний значений этих показателей практически становится сколь угодно большим. А автоматизация процессов организационного управления при решении неструктурированных или слабоструктурированных задач, которые зачастую возникают в управленческой деятельности, представляется весьма сложным в связи с высокой степенью информационной неопределенности. Условием перехода к большей определенности является получение новой информации, что может быть достигнуто с помощью использования СППР [7].

Поэтому на первом этапе создания КСОУ в качестве базовой для СППР составляющей информационной технологии, в работе [5] было предложено использовать ИАСМ. ИАСМ - система специально организованного автоматизированного отслеживания поведения объекта управления, его системы управления и внешней среды их существования по заранее согласованным интегральным показателям с целью определения соответствия их значений 
требуемым прогнозным и плановым параметрам [8-11]. Указанные показатели могут быть использованы в качестве исходных данных в более сложных моделях анализа, прогнозирования и комплексной оценки состояния объекта управления, решении задач повышения эффективности его функционирования с помощью компьютерных моделей.

В зависимости от сложности задач информационной поддержки принятия решений в качестве КСОУ могут применяться информационные системы различного предназначения. Решение же таких задач, как анализ и комплексная оценка обстановки, прогноз изменения обстановки и оценка последствий принимаемых решений, интеллектуальная поддержка пользователей, анализ и прогноз развития внешней экономической и политической ситуации требует создания СППР ориентированных на анализ данных с помощью специальных имитационных, прогнозных, расчетных моделей и средств решения оптимизационных задач.

Очевидно, что в ближайшие годы вряд ли появятся универсальные СППР, реализующие модели функционирования сложных организационно-технических систем, которые можно было бы адаптировать и использовать для управления конкретным объектом. Поэтому, усилия разработчиков систем такого класса будут направлены на создание объектно-ориентированных систем.

Кроме того, на текущий момент не выработано единого общепризнанного определения СППР. Существует некоторое количество определений, раскрывающие отдельные принципиальные отличия подобных систем в множестве автоматизированных информационных систем, связанные с их назначением при использовании в управлении сложным объектом. В нашем случае под СППР будем рассматривать интерактивную объектноориентированную ИАСМ, управляемую данными и моделями, основным назначением которой является удовлетворение информационно-аналитических потребностей лица принимающего решения при решении неструктурированных или слабоструктурированных задач.

Современные информационные технологии позволяют СППР решать следующие классы задач [12]:

- аналитические - вычисление заданных показателей и статистических характеристик функционирования сложных систем на основе ретроспективной информации из базы данных; 
- визуализация данных - наглядное графическое и табличное представление имеющейся информации;

- получения знаний - определение взаимосвязей и взаимозависимостей процессов сложных систем на основе существующей информации;

- имитационные - проведение на ЭВМ экспериментов с математическими моделями, описывающими поведение сложных систем в течение заданного или формируемого интервала времени для анализа возможных последствий принятия того или иного решения.

В результате, процесс создания интерактивной СППР, управляемой данными и моделями, которая ориентирована на решение перечисленных выше задач, необходимо строить на принципах хранилищ данных, к основным из которых отнесены [13]:

1. Разделение данных на показатели (параметры) и измерения, определяющие соответственно состояние и пространство.

2. Логическое представление значений показателей в виде многомерного куба, упорядоченного по равноправным измерениям.

3. Неограниченное число и количество уровней иерархических связей между значениями измерений.

4. Гибкое манипулирование данными, возможность построения подмножества значений показателя по любому правилу, определенному на множестве значений его измерений.

5. Неограниченные возможности агрегирования заданного подмножества значений показателя.

6. Развитые средства табличного и графического представления данных конечному пользователю.

Основная цель хранилищ - создание единого логического представления данных, содержащихся в базе данных (БД).

В настоящей работе, в целях развития и совершенствования ИАСМ ОРЭ, предлагается включить в структуру СППР по управлению функционированием энергорынком следующие компоненты (рис. 1):

- модели объектов, участвующих в структурообразующих связях оптового рынка, определяющих правила его функционирования;

- модели показателей (параметров) функционирования оптового рынка;

- моделирующие алгоритмы (модели различного предназначения), определяющих правила образования и изменения значений показателей функционирования рынка; 
- модель взаимодействия (интерфейса) и представления данных пользователю.

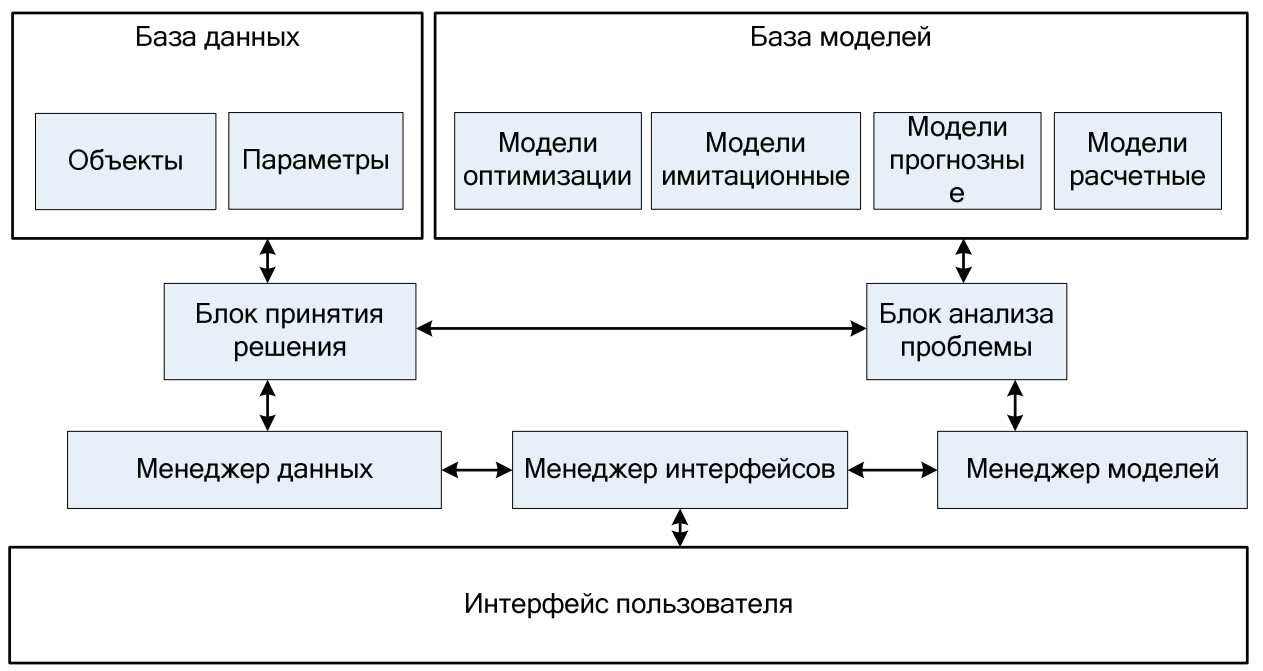

Рис. 1. Концептуальная модель СППР оптового рынка

База моделей в СППР формируется из динамических моделей, в которых анализируемые показатели процессов функционирования энергорынка отображают не только текущее состояние объектов, а и их изменение во времени. Поэтому, в целом база моделей реализуется как трехмерное представление данных в виде куба, где измерениями являются время, объекты, параметры, а ячейками - значения, определяющие состояние процесса функционирования рынка. Такая многомерная модель позволяет делать плоские разрезы куба данных и поворачивать его нужной гранью при поиске затребованной информации разными группами пользователей.

Основными требованиями к моделям СППР служат:

1. Многофункциональность - модель должна позволить проводить расчетные эксперименты в различных режимах (имитационном, прогностическом с проверкой конкретных решений, стратегическом для определения решения, диагностическом для апостериорного анализа и выявления причин наблюдаемых явлений).

2. Представительность - модель должна включать в себя ключевые элементы моделируемой системы (компоненты, факторы, состояния и алгоритмы взаимосвязи между ними).

3. Доступность - модель должна позволять за приемлемое время подготовить и провести расчетные эксперименты.

4. Параметричность - модель должна обеспечивать, при проведении расчетных экспериментов, возможность задания значений требуемого 
множества параметров и начальных условий моделирующих алгоритмов, необходимых для настройки модели по реальным данным.

5. Открытость - возможность доработки и усовершенствования модели.

Требования доступности (универсальности) и представительности (единообразия) в способах представления данных пользователю приводит к необходимости создания так называемых метаданных. Метаданные - это любые данные о данных. Метаданные играют важную роль в построении СППР. Одновременно, их применение является одним из наиболее сложных и недостаточно практически проработанных инструментов в современных информационных технологиях создания автоматизированного управления процессами функционирования сложных систем. В общем случае необходимо выделять такие аспекты метаданных, которые должны присутствовать в системе управления, как с точки зрения описания предметной области, так и функциональности системы:

1) метаданные о структуре данных прикладной области;

2) метаданные о процессах;

3) метаданные о способах представления данных пользователю;

4) метаданные описания пользователей;

5) метаданные по администрированию системы.

Первые два аспекта отражают прикладную специфику решаемых задач и образуют основу модели БД, а остальные - интерфейсную часть взаимодействия пользователя с БД.

Однако, в настоящее время отсутствует единая промышленная технология проектирования, создания и сопровождения метаданных. Поэтому вопросы, связанные с управлением метаданными, следует рассматривать применительно к каждому конкретному проекту построения СППР.

\section{2. Анализ состояния развития информационных технологий проектирования СППР}

Вся история создания КСОУ организационно-техническими системами свидетельствует о постоянном их совершенствовании в плане повышения эффективности использования информационных ресурсов, развития инфраструктуры информатизации, развития применяемых для описания производственных процессов управления математических моделей. Основной движущей силой такого развития является 
продолжающийся практически революционный рост производительности используемых для целей автоматизации средств вычислительной, телекоммуникационной техники и связи, возможностей общесистемных программных средств и средств сетевого взаимодействия конечных пользователей, систем управления базами данных. В этих условиях большое значение приобретает создание прикладных инструментальных программных систем, которые позволяют проектировать КСОУ, отвечающие возрастающим возможностям перечисленных выше средств автоматизации, и обеспечивают решение задач их непрерывного совершенствования.

Современные требования к функциональности КСОУ в сфере мониторинга производственных показателей субъектов ОРЭ (генерирующих компаний, оптовых поставщиков и др.) предусматривают выполнения следующих условий:

- открытость структуры системы;

- гибкость интерфейса для включения новых объектов данных и алгоритмов реализации математических моделей технологического процесса почасового планирования и оптимального распределения объемов производства энергии между производителями и потребителями по критерию минимальной стоимости на производство электроэнергии на заданном интервале графика нагрузки активной мощности;

- прием, обработка, сохранение информации о состоянии субъектов ОРЭ;

- адаптивность к изменениям, как в структуре, так и в значениях параметров субъектов ОРЭ.

Исходя из возможного развития инфраструктуры объекта автоматизации, развития математических моделей, применяемых для описания расчетно-технологических процессов в системе организационного управления, в современных условиях существенное значение приобретает методология проектирования КСОУ с максимально адаптивной и независимой структурой представления данных предметной области от программного обеспечения системы.

Если раньше при создании КСОУ для каждой отдельной функциональной задачи предметной области необходимо было последовательно реализовывать следующие стадии: описание предметной области; проектирование информационной модели (структуры данных) предметной области; разработка логики обработки данных; программирование 
интерфейса системы. То с развитием объектной технологии представления данных [14,15], появилась возможность охватить сложные системы целиком путём проведения их декомпозиции. Кроме того, простое наличие в КСОУ отдельных подсистем еще не обеспечивает решение всего комплекса задач, возникающих как на этапе практического создания системы, так и на этапе ее эксплуатации, связанных с обеспечением надежной и эффективной работы в течение возможно более длительного жизненного цикла.

Идеальным решением проблемы создания адаптивной информационной системы было бы сведение к минимуму модификаций программных модулей системы при переходе к диналическолу лоделированию предметной области объекта автоматизации. При этом структура базы данных и программное обеспечение информационной системы не были бы ориентированы на специфику предметной области и не зависели бы от конкретных прикладных данных.

Однако, распространение принципов объектного моделирования в системах долгосрочного хранения данных, наталкивается на два основных препятствия:

- отсутствие реальных механизмов хранения объектов (в реляционных базах их сохраняют в реляционных структурах и во время работы превращают в объектный вид, что влияет на производительность информационных систем);

- языки программирования не имеют возможности динамично изменять структуру объекта во время выполнения программы.

В этой связи наиболее перспективным направлением в создании КСОУ производственных показателей является подход, основанный на информационной технологии, которая представляет собой гибкое высокоавтоматизированное перенастраиваемое производство моделей автоматизируемых процессов и позволяющее, во-первых, непрерывно совершенствовать технологический процесс создания СППР, поддерживать актуальность инструментальных средств и стандартов, а, во-вторых, что особенно важно, развивать и совершенствовать и саму систему.

Информационная составляющая такой технологии при создании СППР обеспечивает формирование строго определенных структур данных, которые воссоздают модели реализуемых задач и объектов предметной области, учитывают реальные особенности технологических процессов проведения оперативных, плановых и аналитических расчетов и операций по обработке данных. 
Технологическая составляющая технологии представляет собой информационно-технологическую платформу (ИТП), которая обеспечивает создание и функционирование всей совокупности математических моделей расчетно-аналитических задач, логически и информационно взаимосвязанных между собой по целевому назначению, расчетными входными и выходными данными.

В результате логика реализации функциональных задач КСОУ становится элементом данных, представленных в виде собственной модели, которая сохраняется в БД, а основной функцией ИТП становится интерпретация и воссоздание в интерфейсе системы взаимосвязанных данных из внутреннего представления функциональных задач и концептуального представления предметной области объекта автоматизации.

Следует также подчеркнуть, что объектом для разработки в СППР являются не только структуры данных функциональных задач предметной области, но и непосредственно процедуры реализации этих задач. Для этого в ИТП вводится дополнительный логический уровень представления процедурной части прикладной программы. В результате логика реализации функциональных задач становится элементом данных, представленных в виде собственной внутренней модели, которая сохраняется в системе управления базой данных, а основной функцией программного комплекса ИТП становится интерпретация и воссоздание в интерфейсе программы взаимосвязанных данных из внутреннего представления функциональных задач и концептуального представления предметной области.

Таким образом, основной целью предлагаемого подхода является улучшение координации и повышение информационной обеспеченности работы пользователей СППР за счет сокращения сроков подготовки объекта автоматизации, ускорения предоставления интерфейсов взаимодействия пользователя, создания интегрированного информационного ресурса и единой программной среды.

В результате следует выделить следующие направления работ по проектированию и созданию СППР на основе ИТП:

- формирование концептуального представления организационной структуры объектно-ориентированной СППР;

- объектное моделирование данных предметной области с целью формирования единой (неизменной) структуры базы данных субъектов организационного управления; 
- функциональное моделирование технологических процессов обработки данных с целью унификации программного интерфейса системы через типизацию (классификацию) операций взаимодействия пользователя с объектами предметной области в программном обеспечении системы;

- разработка протоколов информационного обмена данными подключаемых моделей различного назначения с базой данных субъектов организационного управления.

\section{3. Организационная структура ИТП построения СППР}

Традиционно при проектировании автоматизированных систем очень часто используется трехуровневая схема построения системы в составе (рис.2):

1) ориентированной на пользователя внешней модели данных уровень представления модулей прикладного программного обеспечения (ПО) функциональных задач информационной системы;

2) концептуальной модели предметной области информационной системы - уровень моделей (структур) данных функциональных задач;

3) физической модели информационных массивов данных - уровень структур базы данных.

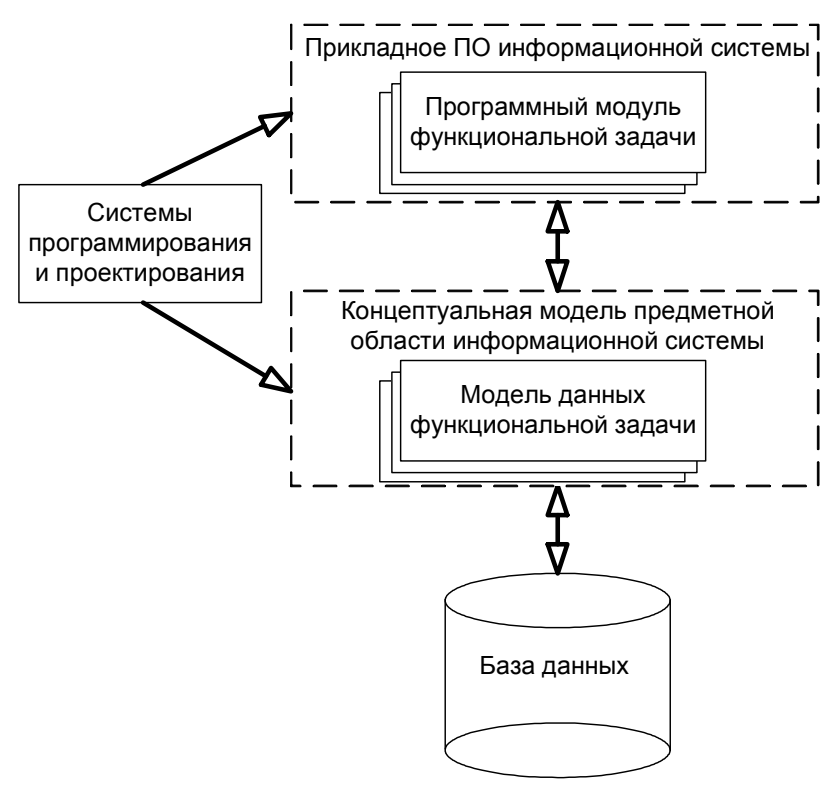

Рис. 2. Статическая структура построения информационной системы

В такой структуре внешняя модель воссоздает способы реализации функциональных задач предметной области, концептуальная модель требования, связи и структуры данных любой из функциональных задач, а физическая модель используется для представления и организации 
доступа к структурированным массивам данных независимо от внутренних свойств предметной области. В концептуальных моделях находят отображения все информационные потоки данных, которые циркулируют в системе, а также функциональные задачи системы, которые выполняют разные операции преобразования потоков, инициированные интерфейсами внешней модели. Концептуальные и внешние модели разрабатываются целенаправленно для задач исследования конкретных проблемных ситуаций. Исходя из данных этих задач устанавливаются границы модели, определяется состав компонент, которые входят в нее, конкретизируется содержание прикладных процессов и способов их анализа. Функции элементов модели определяются в терминах выполняемых им операций преобразования потоков и реализуются в процедурах прикладной программы.

Таким образом, можно говорить о статической структуре организации информационной системы, которая не способна отвечать требованию перехода к диналическолу лоделированию предметной области объекта автоматизации.

Поэтому, в основу гибкой (адаптивной) организационной модели объекта автоматизации должны быть положены типовые черты, которые отражают отличия одного объекта от другого. Целесообразность такого анализа и выделение классов объектов предметной области при разработке внешней и концептуальной модели обусловлена тем, что для разных функциональных задач часто используются одинаковые операции визуализации и преобразования над информационными массивами данных. Кроме того, функции и процессы обработки данных при поэтапном внедрении информационной системы быстро модифицируются, воссоздавая переменные требования пользователей к предметной области. Все это требует такой организации структуры системы, при которой объекты предметной области и взаимосвязи между ними были бы определены независимо от реализации конкретных функциональных задач обработки данных в процедурах и интерфейсах прикладной программы, и представляли собой единую структуру.

В результате, объектом проектирования и описания в организационной модели системы должны выступать не только структуры данных предметной области, а и сами процедуры реализации функциональных задач. Введение дополнительного промежуточного логического уровня представления процедурной и интерфейсной частей программной 
среды ИТП между уровнями концептуальной и внешней модели предметной области обеспечит существенную независимость (адаптивность) СППР от возможных изменений и расширений внешней модели данных (рис.3).

В результате логика реализации функциональных задач предметной области становится данными, представленными в виде собственной модели интерфейса, которая входит в концептуальную модель, а технологической частью ИТП становится инструментальный программный комплекс гибкой (адаптивной) информационной системы, функцией которого служит интерпретация и воспроизведение в задачах системы взаимосвязанных данных из моделей интерфейса функциональных задач и структур данных концептуального представления предметной области.

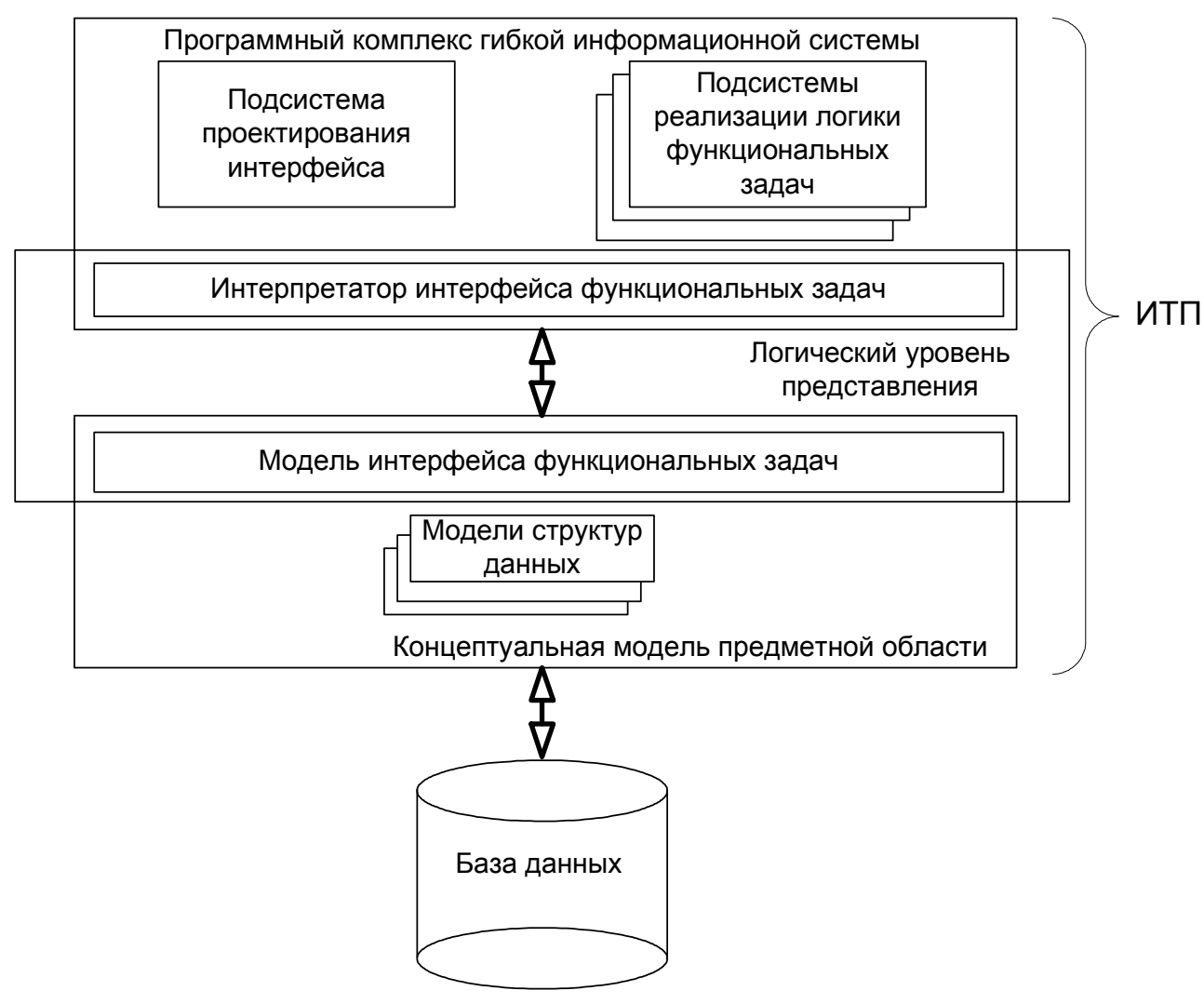

Рис. 3. Организационная структура ИТП построения СППР

Однако простое наличие в информационной системе отдельных функциональных подсистем еще не обеспечивает решение всего комплекса задач, возникающих как на этапе практического создания программной системы, так и на этапе ее эксплуатации, связанных с обеспечением надежной и эффективной работы в течение возможно более длительного жизненного цикла. 
В результате к функциональности современных информационных систем следует предъявлять требования, предусматривающие открытость структуры системы, наличие гибкого интерфейса (механизма) для включения новых объектов и алгоритмов решения прикладных задач и возможности адаптации к изменениям как в структуре, так и в значениях параметров объектов предметной области. Это вызвано стремлением пользователей сократить затраты на сопровождение информационных систем. Поэтому в последнее время наметилась тенденция к проектированию максимально адаптивной и независимой от программного обеспечения структуры хранения данных предметной области в информационной системе. Если ранее при создании информационной системы для каждой отдельной функциональной задачи предметной области необходимо было последовательно реализовывать стадии ее описания, проектирования информационной модели (структуры данных), разработки логики обработки данных, программирования интерфейса системы, то с развитием объектной технологии, появилась возможность охватить сложные системы целиком, проведя их декомпозицию. При этом структура базы данных и клиентское приложение (программа) информационной системы не были бы ориентированы на специфику предметной области и не зависели бы от конкретных данных. В результате, получая информацию из базы, сервер данных заполнялся бы необходимыми алгоритмами обработки, а клиентское приложение строило свой интерфейс при подключении к серверу данных.

В результате информационная (база данных) и технологическая (программное обеспечение) части ИТП будут состоять из функциональной составляющей, отражающей особенности и процессы предметной области, и информационной составляющей, отражающей унифицированные способы взаимодействия с объектами предметной области.

\section{Выводы}

Предложен подход к проектированию объектно-ориентированной СППР, отличительная особенность которого состоит в том, что его программной составляющей становится инструментальный программный комплекс, интерпретирующий представление внутренней модели и реализующий типовые процедуры представления интерфейса в функциональных задачах предметной области. Основу такого комплекса составляет компонентная (модульная) структура реализации типовых функций, на базе которых пользователь решает задачи автоматизации 
процессов управления. Кроме того, компонентная структура воссоздает трехуровневую модель работы с данными, которая предусмотрена в информационной модели программного комплекса. Разработка и применение такой программной среды на предложенной информационнотехнологической платформе дает гарантированное развертывание и сопровождение СППР. И представляет собой единый программный продукт с целью получения максимальных преимуществ от использования открытых систем и в то же время уменьшает риск, сложность и затраты при переходе к автоматизации новых задач управления субъектами энергорынка.

\section{Список использованных источников}

1. Борукаев 3. Х. Модели для определения прогнозной оптовой цены покупки электроэнергии в условиях изменения цен на рынках энергоносителей / Борукаев 3. Х., Остапченко К. Б., Лисовиченко О. И. // Міжвідомчий науково-технічний збірник «Адаптивні системи автоматичного управління» . - 2015. - №2 (27). - С. 35-43.

2. Борукаев 3. X. Моделирование динамики прибыли генерирующих компаний в условиях изменения цен на энергоносители / 3. Х. Борукаев, К. Б. Остапченко, О. И. Лисовиченко // Науково-виробничий журнал Міністерства палива та енергетики України «Енергетика та електрифікація» . - 2015. - №10 (386). - С. 31-35.

3. Борукаев 3. Х. Модель краткосрочного прогноза оптовой цены покупки электроэнергии в условиях изменения цен на рынках энергоносителей / З. Х. Борукаев, К. Б. Остапченко, О.И.Лисовиченко // Міжвідомчий науково-технічний збірник «Адаптивні системи автоматичного управління». - 2016. - №1 (28). - С. 11-22.

4. Борукаев 3. X. Анализ взаимосвязи данных динамики энергорынка с изменениями цен на рынках энергоносителей / З. Х. Борукаев, К. Б. Остапченко, О. И. Лисовиченко // Міжвідомчий науково-технічний збірник «Адаптивні системи автоматичного управління». - 2015. №1 (26). - С. 46-64.

5. Борукаев 3. X. Компьютерные системы организационного управления в энергетике / Борукаев З. Х., Евдокимов В. Ф., Остапченко К. Б., Шатров В. Ф. - Киев: ЦТИ Энергетика и электрификация. - 2002. - 66 с.

6. Поспелов Д. А. Ситуационное управление: Теория и практика. М.: - Наука. - 1986. - 288 с. 
7. Трахтенгери Э. А. Компьютерные системы поддержки принятия управленческих решений // Информационные технологии в управлении: Проблемы управления. - 2003. - № 1, С. 13-28.

8. Борукаев 3. X. Теоретические и информационные аспекты создания компьютерной системы управления топливно-энергетическим комплексом / Борукаев 3. Х., Евдокимов В. Ф., Остапченко К. Б., Шатров В.Ф. // Электронное моделирование. - 2002. - том 24 - № 1. C. 59-70.

9. Борукаев З. X. Об одном подходе к созданию компьютерной системы организационного управления в энергетике / Борукаев 3. Х., Евдокимов В. Ф., Остапченко К. Б. // Энергетика и электрификация. - 2002. №2. - C.49-54.

10. Борукаев З. X. Информационно-аналитическая система мониторинга оптового рынка электроэнергии / Борукаев З. Х., Евдокимов В. Ф., Остапченко К. Б. // Моделирование и информационные технологии. 2002. - Вып. №14. - С.3-13.

11. Евдокилов В. Ф. Некоторые вопросы создания компьютерных средств поддержки принятия решений для систем организационного управления в энергетике / Евдокимов В. Ф., Куцан Ю. Г., Борукаев З. Х., Остапченко К. Б., Грицюк Л. И. // Энергетика и электрификация. 2003. - №1. - C. 2-7.

12. Ларичев О. И. Системы поддержки принятия решений: современное состояние и перспективы развития / Ларичев О. И., Петровский А. Б. // Итоги науки и техники: Техническая кибернетика. - М.: ВИНИТИ, 1987. T. 21, С. 131-164.

13. Apхипенков C. Хранилища данных. От концепции до внедрения / С. Архипенков, Д. Голубев, О. Максименко, Под общей редакцией С. Архипенкова. - М. : ДИАЛОГ - МИФИ, 2002. - 528 с.

14. Грэхэл И. Объектно-ориентированное моделирование. Принципы и практика. М. : Изд. - во - Вильямс, 2004. - 880 с.

15. Буч Г. Объектно-ориентированный анализ и проектирование с примерами приложений на $\mathrm{C}++$. Третье изд. Пер. с англ. И. Романовского, Ф. Андреева. М. : Изд. - во - Вильямс, 2008. - 720 с. 